\title{
Langue et scolarisation dans une communauté asiatique en France : les Hmong
}

Kao-Ly Yang

\section{(2) OpenEdition}

1 Journals

Édition électronique

URL : http://journals.openedition.org/trema/2195

DOI : $10.4000 /$ trema.2195

ISSN : 2107-0997

Éditeur

Faculté d'Éducation de l'université de Montpellier

\section{Édition imprimée}

Date de publication : 1 juin 1995

Pagination : 65-73

ISSN : 1167-315X

\section{Référence électronique}

Kao-Ly Yang, «Lanque et scolarisation dans une communauté asiatique en France : les Hmong »,

Tréma [En ligne], 7 | 1995, mis en ligne le 23 septembre 2013, consulté le 30 avril 2019. URL : http:// journals.openedition.org/trema/2195 ; DOI : 10.4000/trema.2195

Ce document a été généré automatiquement le 30 avril 2019

Trema 


\title{
Langue et scolarisation dans une communauté asiatique en France : les Hmong
}

\author{
Kao-Ly Yang
}

1 Cette étude sur les difficultés de l'apprentissage du français chez des enfants Hmong de Nîmes, originaires du Laos, sera centrée en particulier sur la mise en rapport des langues hmong et française et la représentation du savoir.

2 Les Hmong, exilés en France, vivent une situation de contact de langues avec le français. La population Hmong est arrivée récemment en France, à la fin des années 70 : leur implantation sur le sol français est récente, une quinzaine d'années. C'est une durée très brève dans l'échelle du temps d'acculturation. Pour eux, c'est une période marquée par des emprunts ou appropriations de traits culturels, signes de la mise en jeu de leur langue, de leur culture, de leurs traditions... Cela ne se fait pas sans confrontations ou même sans conflits. A long terme, les modes de perpétuation ou transmission de leurs us et coutumes à leurs descendants dans la société française, peuvent être, sous l'effet de l'acculturation formelle, changés ou perdus.

Depuis toujours, les Hmong sont des ruraux. Et toutes leurs traditions sont transmises oralement, ce qui ne facilite pas à priori leur intégration dans une société urbaine et fondée sur l'écrit. Leur rapport à l'écriture est intermittent car il ne s'active que durant les révoltes. A ces périodes de trouble, une certaine écriture ou niam mtawv apparaît ou réapparait comme attribut d'investiture divine qui aurait le pouvoir de changer leur destin de peuple sans royaume en une nation avec territoire, roi et écriture. Ces faits historiques mettent en évidence un rapport très mythifié à l'écrit. Ce qui de nouveau ne les rapproche pas des sociétés aux traditions scripturales anciennes.

Dans ce contexte de réfugiés politiques en France, on se posera la question de savoir comment ils vont négocier leur entrée dans la société d'accueil, notamment le passage de l'oral à l'écrit. Ce qui fait la nouveauté de la situation, c'est la nécessité et l'obligation d'emprunter les canaux de l'enseignement et de la communication. En Chine, au Laos, ils pouvaient vivre en vase clos dans les montagnes. Ce n'est pas le cas en France. 
On posera comme hypothèses que :

- il y a évolution de la langue hmong, liée à celle du rapport de l'oral avec l'écrit et la connaissance de fonds socioculturel français et hmong (traditions, chants) et linguistique en hmong (lexique) ;

- l'école est le seul lieu de rencontre avec l'écrit ;

- la représentation du savoir régit les choix des enfants en ce qui concerne les orientations scolaires et professionnelles.

\section{Qui sont les Hmong?}

6 C'est un peuple qui fait partie de la mosaïque ethnique de Chine. Leur origine géographique est localisée dans le bassin fluvial des fleuves chinois Huang Ho et YangTseu. L'histoire des Hmong est faite de fuites, d'exil. En effet, au XIX siècle, beaucoup d'entre eux ont pris la fuite à la suite de la violente répression chinoise : ils se sont installés en Birmanie, en Thaillande, au Laos et au Vietnam du Nord. A la suite d'une implication massive des Hmong surtout au Laos dans les guerres d'Indochine aux côtés des Français puis des Américains, et la chute du gouvernement royal en 1975, ils connaissent de nouveau l'exil : cette fois-ci, après des séjours dans les camps de réfugiés en Thailande, ils se retrouvent dans les pays de l'Occident (USA : 100 000, France $: 7000$ et Guyane française : 1 000, Australie : 2 000, Canada : 2000 ).

\subsection{L'organisation sociale et familiale et le modèle éducatif}

7 Le modèle éducatif hmong est lié à la famille et au clan (groupe ayant un ancêtre réel ou mythique en commun), car les Hmong vivent en clan et ils sont profondément attachés à cette appartenance qui est une marque d'identité. L'enfant porte le nom du clan du père. La cohésion de la société hmong est basée sur l'échange de membres féminins par le mariage - ce qui étend les relations familiales aux autres clans et explique que dans les moments importants de la vie hmong (la mort et le mariage), la solidarité se manifeste comme le signe d'appartenance et d'alliance clanique servant de garantie de cette cohésion. Certains ethnologues ${ }^{1}$ qualifient cette organisation sociale d'égalitaire: les critères d'observation de cet égalitarisme sont notamment les termes d'adresse qui ne marquent ni l'infériorité ni la supériorité des membres, terminologie qui est le reflet des structures sociales. En ce qui concerne l'organisation familiale, il y a prédominance des hommes et la division du travail respecte le rôle de l'homme et celui de la femme dans cette société traditionnelle.

8 Le père détient l'autorité en matière d'éducation et prend les décisions (mariage, études, etc.). La mère a un rôle secondaire et sert d'intermédiaire auprès de son mari et de ses enfants. C'est elle qui concrètement s'occupe des tâches administratives et ménagères avec la participation de sa fille aînée. A Nîmes, 14 familles (sur les 16 de cette étude) ont des activités agricoles, qui donnent aux parents une image valorisante auprès de leurs enfants et offrent une possibilité d'entrer dans la vie active aux enfants peu doués à l'école.

9 L'éducation des filles vise à former de bonnes épouses et mères au foyer. A l'âge nubile, elles sont initiées aux préparatifs du mariage; en France, cela se traduit par une autorisation d'aller dans les fêtes hmong à partir de 13 ans. Les qualités principales de la 
femme sont de savoir parler et de recevoir les hôtes. Ses qualités intellectuelles sont peu valorisées.

L'éducation des garçons comporte moins de contraintes (plus de libertés de sortie). Le mariage peut être repoussé. Le savoir parler reste un trait d'éducation et les connaissances intellectuelles contribuent socialement à les valoriser.

11 On remarquera que cette éducation est donnée par les autres membres claniques (maternels y compris). En effet le clan est :

- le point de repère de l'éducation : interdiction de se marier entre membres d'un même clan ; les filles se marient pour devenir membre reconnu socialement d'un autre clan; les garçons restent dans le clan et maintiennent le flambeau des traditions ; on encourage davantage les garçons à fréquenter l'école car ils ne sortent pas du clan contrairement aux filles...

- un point de renforcement éducatif : les affinités qui existent entre fille et belle-sœur, fille et tante, fils et beau-frère, fils et oncle permettent de transmettre des pratiques traditionnelles («drague », éducation sexuelle, chants, broderie...)

- le point d'ancrage de la réussite : le clan confirme et reconnaît les succès car la réussite individuelle n'a pas de sens en elle-même.

\subsection{Les Hmongs et l'écriture}

La langue Hmong est une langue tonale, appartenant à la famille Miao-yao ou Hmongmien. Deux dialectes parmi les sept en Chine se sont retrouvés au Lao : le Hmong Blanc et le Hmong vert, appelés ainsi d'après la couleur des jupes des femmes.

Les Hmong ne disposent pas d'une écriture propre, existant depuis longtemps et commune à tous les Hmong dispersés à travers le monde. Certes, certaines populations hmong ont inventé des écritures à un moment donné de leur histoire. Mais ces écritures n'ont jamais réussi à s'imposer ni comme un élément réunificateur des Hmong ni comme outil d'échange entre eux. Une cause possible vient des conditions d'émergence: ces inventions n'ont lieu que pendant leurs révoltes à caractère messianique. Et ces écritures ne durent que tant que les révoltes durent, car elles sont la marque d'investiture divine pour légitimer ces mouvements révolutionnaires; une fois la révolte passée, l'écriture demeure l'objet de souvenir, réservée à quelques adeptes seulement. Mais cette absence est vécue d'une façon complexée par les Hmong vis-à-vis des populations qui ont une tradition de l'écrit, malgré les transcriptions du hmong qui existent en plusieurs versions (à caractères chinois, thaïlandais, laotiens et latins).

14 Ainsi les Hmong ont à la fois une relation mythique avec l'écriture, représentée comme magique, et une représentation formelle et réductrice de l'écrit appréhendé comme caractère, lettre. En effet, pour eux, apprendre dans le cadre de l'école, lieu du savoir, c'est avant tout apprendre à lire et à écrire, c'est à dire connaître la lettre : en hmong, on dit " paubntawv " (savoir lire et écrire) ou " mus kawv ntawv » (aller à l'école) (paub : savoir, ntawv : lettre (s), caractère, écrit, écriture, missive). 


\section{L'enquête}

\subsection{Les caractéristiques de la population d'étude}

Il s'agit de la communauté Hmong nîmoise, habitant les Zup Nord et Sud. Ces deux quartiers sont caractérisés par une population issue de plusieurs origines géographiques et culturelles (américaine du Sud, arabe, asiatique, européenne de l'Est et du Sud). Le chômage touche une moyenne de 27 \% de la population globale. De même l'échec scolaire concerne $43 \%$ des collégiens.

La présence des Hmong dans ces deux quartiers date de l'année 1979. Actuellement, il y a une centaine de familles qui se sont regroupées à Nîmes, à la fin des années 1980, qui rassemblent 900 personnes environ.

\subsection{L'échantillon}

17 Il représente 5,5\% de la population hmong de Nîmes, soit les 16 familles les plus tôt implantées à Nîmes. Leur origine géographique au Laos est située autour de la plaine des Jarres (province de Xieng Khouang) où il $\mathrm{y}$ a eu les premiers et les plus importants contacts linguistiques et culturels avec les Laotiens et les Français. Là-bas, ils étaient de petits producteurs terriens. Ces 16 familles dont une est polygame ont 130 enfants. Pour appréhender les représentations de l'école et des usages des langues hmong et française chez les enfants Hmong, 51 des 130 ont été choisis suivant le critère du niveau scolaire : être entre le cours élémentaire deuxième année (CE2) et la seconde (en filière technique ou non); la tranche d'âge s'étale donc de 8 à 18 ans. Signalons que 32 enfants des 51 sont nés en France et les 19 autres avaient en moyenne 2 ans à leur arrivée.

\subsection{L'approche méthodologique}

Une pré-enquête sous forme de discussion libre et d'observation a été menée pour délimiter les centres d'intérêt et les lieux de parole (maison, école, quartier) ce qui a permis d'établir un questionnaire destiné aux parents portant sur la scolarité de leurs enfants en France et au Laos. On a utilisé des interviews directives (dialogues) ou non directives (récits de vie). Un second questionnaire a été établi pour les 51 enfants. Enfin, des interviews directives ont lieu avec les autres enfants pour connaître leur scolarité et leur situation professionnelle.

19 Cette approche cherche surtout à quantifier et à objectiver les réponses et les représentations concernant l'école et les pratiques langagières. Une enquête menée par une Hmong sur sa propre communauté, dans un milieu familier, soulève le problème de l'objectivité, étant données les habitudes langagières et les représentations établies. Ainsi, dans la collecte des informations et dans leur traitement, on se base sur « l'idée qu'il existe une différence spécifique entre la représentation subjective et l'objet qu'elle reflète $»^{2}$. De plus, le rôle et le statut de l'enquêtrice sont ici doubles :

- elle est l'enquêtrice indigène soumise au regard des siens. Ce rôle d'enquêteur est inhabituel pour les Hmong car cette activité n'existe pas chez eux. Durant l'enquête, les parents réintègrent une représentation habituelle d'une jeune fille hmong et d'une étudiante : elle doit éduquer les enfants (i.e. enseigner aux enfants) et elle doit faire connaître les Hmong à 
travers sa recherche. Elle a un rôle d'intermédiaire mais non celui du porte-parole (celui-ci est réservé aux hommes) ;

- elle est aussi l'enquêtrice migrante qui porte un regard sur les siens. C'est un aspect lié à la rencontre culturelle spécifique qu'elle a faite avec la société d'accueil. Son processus d'acculturation peut alors modifier son approche dans ses démarches et dans les termes utilisés (méthodologique, ...) pour cerner la réalité de l'école par exemple. Ces termes peuvent être hmong ou français.

\section{Analyse de l'enquête}

français à l'oral, mélange qui sert d'outil de communication entre enfants hmongs, et aussi entre ces derniers et leurs parents. Il est perçu comme langue hmong enrichie de mots français par exemple : «lub poche to gov " (la poche est trouée) ou " peb lub-télépuas lawm » (notre télé est cassée). Il s'agit d'une substitution lexicale; les "articles » hmong sont conservés, ce qui marque un même rapport perceptif aux formes des objets ou choses ; ainsi, la langue garde sa vision du monde. L'importance de la pénétration de mots français dans le hmong est due peut-être au fonctionnement de la langue orale qui privilégie l'efficacité et l'économie de l'échange verbal. De plus on trouve dans ce mélange un emploi fréquent des conjonctions de coordination ou de subordination françaises, qui remplacent ou s'ajoutent à celles du hmong ${ }^{3}$. Cette présence est peut-être liée à une interférence de structures syntaxiques. Car à travers les questionnaires, un tiers des enfants affirment avoir à la fois des difficultés à faire des phrases et à s'exprimer en hmong ; l'observation montre qu'ils se figent sur des formes structurales de la syntaxe française à l'oral comme support d'énonciation. Ainsi, ces difficultés seraient dues à une confusion entre le système de l'oral et celui de l'écrit : ils réduisent formellement les complexités énonciatives de la langue hmong en complexité formelle et syntaxique. D'une part, cela suppose une mise en rapport du hmong avec le français au niveau du système écrit avec un transfert de structures syntaxiques à la langue maternelle. D'autre part, la perception du sens à l'oral soit à l'aide des mots ou à l'aide des fonctions syntaxiques tient plus d'un mode de l'écrit que de l'oral; ceci montre une inscription formelle de la part des enfants dans le schéma de l'écrit au détriment de celui de l'oral. Toutefois, il s'agit d'une influence du français de l'école, représenté par les enfants comme le français de l'écrit.

Par ailleurs, les trois grandes difficultés apparues dans l'enquête (manque lexical, incompréhension orale, construction de phrase) apportent un éclairage à des problèmes de transmission culturelle : la réduction formelle des complexités énonciatives est liée à 
une méconnaissance du fonds lexical hmong et certainement à une interférence des schémas oral et écrit. De plus, l'absence de fonds socioculturel français chez les enfants est un facteur d'inégalité dans cette mise en rapport: il y a aussi méconnaissance ou ignorance des pré-requis sociaux français. En effet, en se focalisant sur les formes syntaxiques, ils ont un rapport figé à la structure sans pouvoir la dépasser pour accéder aux savoirs, à la culture d'accueil qui leur permettront la promotion sociale. Ainsi, des difficultés peuvent apparaître au niveau du second degré où le facteur socioculturel est un élément déterminant pour la réussite. Le rapport récent de cette communauté avec l'écrit entraîne des confusions et empêche une véritable appropriation.

Enfin se pose le problème du français de l'école. Il y a une mise en rapport à l'oral du $\mathrm{hmong}$ et $\mathrm{du}$ français populaire. Pour favoriser la réussite scolaire des enfants et se faire comprendre d'eux, les parents n'hésitent pas à employer un français populaire mélangé au hmong quand ils s'adressent à leurs enfants. Cette pratique langagière ne correspond pas à celle de l'école et pourrait donc être un obstacle à l'apprentissage du français standard, car elle crée une confusion de registres de langue dépendant du lieu de parole. Ceci apparaît dans le questionnaire : environ $40 \%$ des enfants ne comprennent pas «ce que dit le maître ».

\subsection{La représentation du savoir comme facteur des orientations scolaires}

\subsubsection{L'école et l'enseignant}

Depuis la Chine, les Hmong ont très peu affaire à l'enseignement. Les Hmong ont occupé que ce soit en Chine ou au Laos, les régions montagnardes et éloignées des villes. Au Laos, où ils sont arrivés à la moitié du XIX siècle, la communauté hmong a accédé à l'école seulement dans les années 1940. Ce n'est que vers 1970 qu'on observe l'émergence d'un petit groupe d'étudiants en Occident. Ce petit nombre d'élèves devait acquérir un savoir rudimentaire d'écriture et de lecture pour l'échange entre communautés ethniques. Ceci favorise une représentation de l'école comme le lieu de prestige permettant une ascension sociale. L'enseignant garde l'image du détenteur de savoirs car il possède l'écriture.

Notre présente enquête sur les Hmong de Nîmes révèle les mêmes représentations de l'école et de l'enseignant; cela persiste malgré la gratuité et l'obligation scolaires pour tous. Ainsi, la représentation du savoir peut être un facteur déterminant des choix d'orientation scolaire et professionnelle chez les Hmong. Il faut tenir compte de l'organisation sociale et familiale qui régente l'éducation des enfants et exerce toujours son autorité sur ses membres : elle influence les choix professionnels. De plus, la situation particulière de réfugié est aussi un autre facteur important: les Hmong ont à appréhender de nouvelles situations et pour cela, ils préfèrent celles qui leur sont familières et connues. Ils doivent aussi travailler pour subvenir à leurs besoins. Et les cycles courts correspondent mieux à leurs attentes.

\subsubsection{La représentation du savoir}

Elle revêt un caractère oral car ce savoir sert avant tout l'éducation des Hmong : le savoir vivre est basé sur le savoir parler (connaître les règles de bienséance hmong). Savoir parler apparaît en effet comme bien plus important que savoir écrire et savoir lire. La 
parole a valeur d'acte : elle peut blesser, entraîner des discordes, des fautes dans les pourparlers, lors d'un mariage par exemple. Les Hmong exigent réparation dans ces caslà sous forme de dédommagement financier... Les Hmong n'ayant pas de textes de lois écrits, c'est la mémoire collective constituée de toutes les paroles qui ont été dites qui sert de base de référence pour régler les futurs conflits. Cette mémoire joue un rôle important dans la cohésion judiciaire et sociale.

Par ailleurs, l'organisation du savoir chez les Hmong est la juxtaposition de deux types de savoirs: les savoirs pratiques ou traditionnels et les savoirs abstraits ou acculturés (car empruntés à d'autres peuples). Ce sont deux réseaux de connaissances parallèles. La première catégorie concerne plutôt les savoirs pratiques ou techniques incluant les savoirs-faire, par exemple « paub + ua teb» (savoir travailler au champ) ou " paub + ntaus blau » (savoir forger le fer). La deuxième catégorie va de la magie jusqu'aux sciences et comporte des savoirs en provenance de l'étranger, exprimée dans le terme précis «txuj $c i$ » (science au sens très large), dont la particule «ci » signifie «brillant», à prendre peut-être dans le sens d'«attrayant» et "supérieur ». L'écriture, que les Hmong ne possèdent pas, appartient à cette seconde catégorie. Car elle est pour eux le sceau du savoir et l'accès à la modernité.

\subsubsection{Savoir et orientation scolaire}

29 A partir des métiers envisagés et exercés par les enfants, une configuration des savoirs est établie. Il y a double mise en correspondance : celle des «txuj ci» avec le domaine des sciences et celle des savoirs traditionnels hmong avec les savoirs pratiques et techniques français, lesquels ne nécessitent pas en général de longues études : cycles courts après la 5 ${ }^{\mathrm{e}}$ ou la $3^{\mathrm{e}}$ ou cycles techniques. Les cycles courts ont une traduction en hmong : «kawm ua bauj lwm» (apprendre à travailler). En général, un tel choix est envisagé du fait que l'enfant (le fils) doit prendre ses parents en charge (si ces derniers sont devenus inaptes au travail) ou du fait qu'il n'a pas les moyens intellectuels, socioculturels nécessaires pour continuer.

Mais ces enfants ont une relation positive avec l'école : l'échec scolaire n'est pas vécu de façon dramatique car l'école de la vie leur semble tout aussi bonne pour se former. En effet, ces choix d'orientation entrent dans leur représentation du savoir et de son organisation : c'est une réussite pour eux, quand ils ont une possibilité de gagner leur vie dans leur situation de réfugiés. De plus les réussites concernent les domaines techniques et demeurent individuelles et isolées. Pour que l'école soit une chance véritable pour tous, le rapport figé et formel que les Hmong entretiennent avec l'écrit doit être dépassé : apprendre à écrire n'est pas apprendre à faire une rédaction pour avoir une bonne note mais prendre possession de l'écrit comme mode de vie.

\section{Conclusion}

31 Cette étude s'est efforcée de montrer en quoi la communauté Hmong en France vivait une situation spécifique de langue seconde. En effet, avec les regroupements familiaux dans les quartiers souvent classés comme défavorisés, il est possible que cette communauté connaisse une situation d'intégration identique à celle des migrants: en matière de réussite scolaire, il y a superposition de la difficulté économique et socioculturelle à leur problème spécifique de rapport à l'écrit. On pourrait toutefois voir apparaître des 
problèmes plus préoccupants avec la deuxième génération qui fera son entrée dans le monde du travail : les orientations en cycles courts seront certainement un handicap à une réussite d'insertion. Mais le rapport dialectique avec l'écrit laisse entrevoir une relation favorable et positive avec l'école, ce qui permet de supposer une acquisition progressive des pratiques de l'écrit qui constituera un tremplin à l'intégration en France.

Un lycée en Indochine

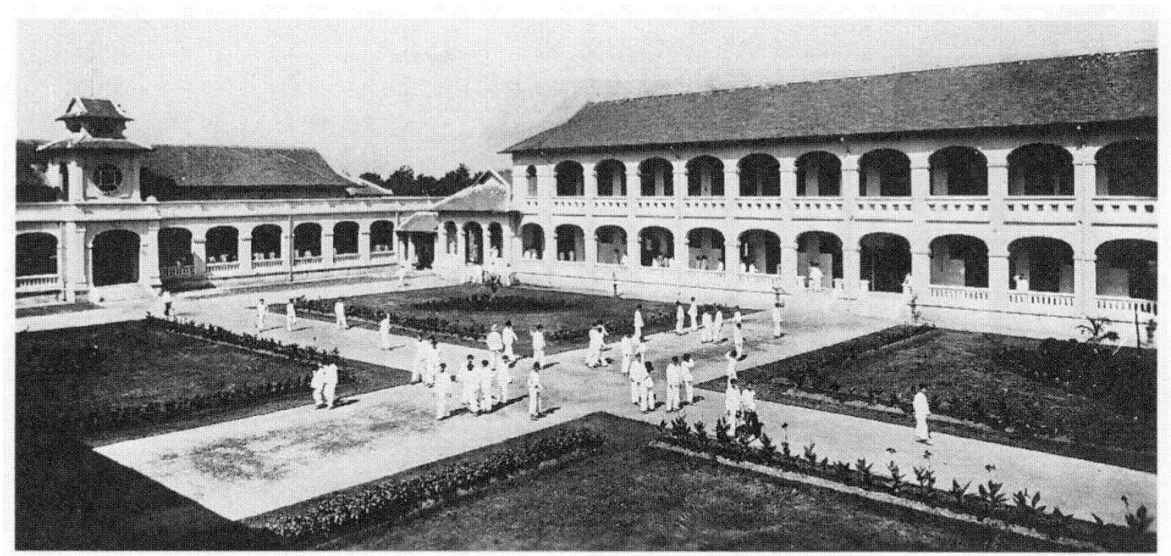

\section{NOTES}

1. HASSOUN J.-P., « Les Hmong à l'usine ", Revue française de sociologie, vol XXIX, Paris.

2. MARCELLESI J.B., GARDIN B., Introduction à la sociolinguistique, Paris, Larousse, 1974, p. 248.

3. MOTTIN J., Eléments de grammaire de Hmong blanc, Bangkok.

\section{RÉSUMÉS}

Une communauté Hmong, originaire du Laos, est installée en France depuis une quinzaine d'années. L'enquête porte sur un échantillon de cette communauté habitant dans la ZUP de Nîmes. Elle étudie les particularités de la scolarisation des enfants Hmong, notamment le modèle éducatif, les modalités de l'accès à l'écrit et les représentations du savoir.

A Hmong ethnic group originating from Laos, is established in France for some fifteen years now. This investigation bears upon a population sampling of members of that group living in the community social housing project (ZUP) of Mmes. This investigation bears upon what is spec ally different in the instructing of Hmong children, notably their society's educational model, its ways and means of acceding to the written word and its representation of knowledge. 
INDEX

Mots-clés : acculturation, écriture, hmong, scolarisation

Keywords : acculturation, hmong, process of instruction, written word

\section{AUTEUR}

\section{KAO-LY YANG}

Etudiante en maîtrise de Sciences du Langage à l'université de Montpellier III 